\title{
Applying Internet Geographic Information System for Water Quality Monitoring
}

Piotr Jankowski*, Ming-Hsiang Tsou and Richard D. Wright Department of Geography, San Diego State University

\section{Abstract}

This article explains the fundamental concepts of Internet geographic information system (GIS) technology and illustrates its capabilities using the example of water quality monitoring in San Diego Bay Watershed, California. Internet GIS technology has democratized public access to geographic data and information services, and software applications such as Google Earth provide millions of users with instantaneous access to digital geographic data and tools that, until recently, were only available to GIS specialists. In addition to making geographic data accessible to a large number of potential users, Internet GIS is also a technological vehicle for encouraging public participation in local decision-making processes. Key developments of Internet GIS technology are highlighted. The links between Internet GIS and public participation are summarized and an application called Common Ground is described. Common Ground delivers data and information about water quality in the San Diego Bay Watershed to the public. The design, architecture, and capabilities of this Internet GIS application are discussed, and the lessons learned and the outlooks for future Internet GIS developments are evaluated.

\section{Introduction}

The development of Internet geographic information system (GIS) dates to the mid-1990s, and is the direct consequence of the development of Internet and Web technologies. It is fair to state that Internet GIS has brought geospatial data and services to the Internet-literate masses. Perhaps the most spectacular statistic illustrating this fact is the number of installations of Google Earth, the popular virtual globe data viewer, which surpassed 200 million at the beginning of 2007. Yet, the vast majority of Google Earth users have never even heard of GIS. However, this has not stopped them from using geographical data in a variety of ways creating communities of users. Geospatial technology infrastructure developed originally by GIS professionals, because GIS professionals are now in the hands of plural publics due to the expansion of the Internet.

Today, Internet GIS applications enable access to current data through simple to use Web-based map viewers. The basic capabilities of the viewers 
include navigating around a map and querying an application database, which usually resides on one or multiple server computers connected to the viewers through the Internet. The applications of Internet GIS are located in many domains, including commercial, government, research, education, and private use. Their common characteristic is the separation of the database from the data viewer and consequently from data users. Such a separation enables a flexible access to data from different locations at different times. A few examples include organization-wide GIS databases (e.g. an electric utility company database about locations and characteristics of power line equipment), weather maps, ocean current maps, real estate property maps, address locator maps, dynamic traffic maps, and assessor parcel databases made available to the public through Internet GIS data viewers.

The intent of this article is to present an overview of Internet GIS technology, and illustrate its capabilities using the example of Common Ground - an Internet GIS Web portal providing access to water quality data about San Diego Bay Watershed and its geospatial functionalities. The choice of application domain, water, is not coincidental. Water resources play a vital role in the well-being of communities worldwide and they cut across a number of economic, environmental, individual, and legal interests. In the San Diego Bay Watershed, for example, there are multiple cities and agencies in charge of monitoring, managing, and distributing water to its end-users. These various local government entities almost continuously collect data about water quality, storing them in their respective databases. Up until recently, sharing the data among these entities has been difficult at best. Because water quality data are sampled at specific locations, the coordinates of each sample can be stored in GIS databases along with water quality sample characteristics. Such data, if collected from all of the entities engaged in water quality sampling, can be made available to all potential users through an Internet GIS application.

We begin the article by explaining what Internet GIS is and what its main components are. Next, we introduce the concept of participatory GIS, and explain how Internet GIS can facilitate public access to data, information services, and develop skills necessary for meaningful public involvement in the stewardship of aquatic resources. In order to ground our discussion in the reality of water quality management in the United States, we explain the meaning and function of Total Maximum Daily Loads (TMDL) in establishing water quality monitoring. We then present the design of Internet GIS for water quality monitoring in San Diego Bay Watershed discussing its objectives, design process, and functional capabilities. We also present an evaluation of the portal usability based on a small sample of questionnaires given to a selected group of portal users. We conclude the chapter with a brief account of lessons learned during the design and implementation of Common Ground, and give our vision of future Internet GIS based on distributed, deliberative-analytic geoservices. 


\section{What Is Internet GIS?}

Internet GIS is the cyber-infrastructure framework for geographic information services (GIServices) utilizing both wired and wireless Internet to access geographic data and spatial analytical tools. Internet GIS is different from traditional geographic information systems (GISystems) in that GIServices focus on open, distributed, task-centered information services, which broaden geographic information uses into an increasingly wider range of online geospatial applications. Where GISystems are functionoriented, geographic information sciences (GIService) are user and serviceoriented. Both GISystems and GIServices are part of the research domain in GIScience, which provides fundamental scientific theories and technologies for both GISystems and GIServices. Early Internet GIS prototypes appeared in the mid-1990s. Xerox map viewers, the Alexandria Digital Library project, and GrassLinks are a few representative Internet GIS prototypes from the early period of Internet GIS (Buttenfield and Goodchild 1996; Huse 1995; Plewe 1997; Putz 1994). More recently, we have witnessed an explosion of interest in transforming GIS into the ubiquitous, distributed, Internet GIServices. These services are of value not only to various professionals using geospatial data in their daily work, but also to the general public using spatial information for navigational and general information purposes involving location-based data. There are Web sites powered by Internet GIS that allow people to check city zoning and parcel information, to see where and what types of crimes are occurring in their neighborhoods, and to learn when the trash pick-up day on their street is (Peng and Tsou 2003).

Geographic information system on the Internet presents a compelling and effective way of disseminating spatial information when compared with traditional paper maps or digital maps stored in centralized GISystems (Tsou 2004). Although the melding of GIS and the Internet seems commonplace now, the idea was revolutionary in 1993. One of the earliest Internet GIS prototypes called Xerox PARC map viewer appeared on the World Wide Web (WWW) in 1993 (Putz 1994). The map viewer, developed at the Xerox Corporation Palo Alto Research Center, allowed users to zoom in on a location on the picture-based map rendering of the globe, and retrieved a map of the selected area from a geographic database. In 1994, two important organizations promoting standardization in Internet GIS were established; the Open Geospatial Consortium (OGC) and the International Organization for Standardization (ISO) Technical Committee 211 (TC211). Specifications developed by OGC and ISO/TC211 for spatial data formats, data exchange, and data communication have become the bases for software interoperability and the on-going development of Internet GIS (Green and Bossomaier 2002; Peng and Tsou 2003).

Another development milestone for Internet GIS was the launch of Google Earth in June 28, 2005 by Google Inc., after the company 
acquired Keyhole Corporation and its popular viewer, EarthViewer 3D (source: www.google.com/press/pressrel/keyhole.html, last accessed on July 27, 2007). Google Earth is a revised version of EarthViewer 3D. The powerful three-dimensional visualization capability, detailed satellite imagery coverage, and innovative user interfaces have made Google Earth the most popular Internet GIS application ever. Many scientists have started to utilize Google Earth in their research as a visualization and exploratory data analysis tool. Users can also add their own geospatial data into Google Earth by generating Keyhole Markup Language (KML) files and linking multimedia components, such as videos or Web pages. Many federal and local government agencies have started also to explore the possibility of using Google Earth for variety of tasks including environmental monitoring, conservation, and homeland security. As Declan Butler wrote in his lead article published in Nature, February, 2006,

One of the traditional roles of GIS has been to provide data to support decision-making. And environmental groups that have discovered GIS are starting to use it to change the balance of power in public debates. As more citizens become concerned about their local environment, easy-to-use virtual globes will facilitate the communication of spatial information between stakeholders and government agencies. (Butler 2006, 778)

In the area of cartography, Internet GIS also provides a revolutionary approach to disseminating geospatial information in various map formats, such as Web Mapping Services (WMS) or multimedia presentations (Peterson 2003). Internet GIS has had a significant impact on map making and the delivery of maps to their end-users. Innovative digital representation methods, such as the virtual globes displayed in the Google Earth, ArcGIS Explorer, and Microsoft Virtual Earth, illustrate a new direction for modern digital cartography. With new Web-based mapping technologies and Internet GIS tools cartographers and mapping scientists can focus on the scientific explanations and mechanisms of geospatial information communication. Future developments in cartography will focus on the effectiveness of new user interfaces, such as touch table screens, speech recognition tools, or motion detection sensors, as well as explore the scientific meanings of the mapping language in various formats. Recent Web technologies such as Asynchronous JavaScript and XML (AJAX) and image tilting is this a list of things, it should be comma spaced if so, or not? Yang et al. (2005) have improved already the performance of Web map servers and made Internet GIS more usable and appealing.

Internet GIS is a paradigm shift in the development of GIS. In order for GIS functions and services to work properly, most Internet GIS applications utilize open and interoperable computing environments and protocols, including WWW, File Transfer Protocol, and Z39.50 protocol. They also utilize distributed programming languages, including Java, JavaScript, Python, or C-sharp, to connect multiple user machines and servers. 
Internet GIS derives from the concept of distributed system architecture. The most significant difference between traditional GISystems and Internet GIS is the adoption of distributed component technology and distributed computing languages, which can be used to access and interact with multiple and heterogeneous systems and platforms (Huang and Worboys 2001; Plewe 1997; Vckovski 1998). Internet GIS architecture permits dynamic combinations and linkages of geodata objects and GIS programs via networking (Peng and Tsou 2003). The technology enabling the Internet GIS uses information networks connected through communication devices and network protocols. The WWW is a networking application supporting a HyperText Transfer Protocol (HTTP) that operates on top of the Internet. Hence, Internet GIS is not synonymous with Web GIS. Internet GIS refers to using a wide-area network to distribute typical GIS tasks including data editing, analysis, and visualization, whereas Web-based GIS refers specifically to the use of WWW to carry out GIS tasks. For example, Google Earth and Environmental Systems Research Institute (ESRI) ArcGIS Explorer are powerful Internet GIS applications, but they are not Web GIS because these software packages are not running inside Web browsers. Conversely, Google Maps and ArcIMS viewers are Web GIS applications because they do need a Web browser to display maps. The advantage of Web GIS applications is that they are more accessible because most computers have built in Web browsers. Some Internet GIS applications require local installations of map viewers (such as Google Earth), which might be difficult to accomplish on publicly used computers, such as in a public library. As a potential disadvantage, Web GIS might be limited in GIS functions and capabilities because of the limitations of Web browsers. Internet GIS applications are usually more powerful and customizable than Web GIS applications.

Interoperability is the key issue for the establishment of Internet GIS, because distributed hardware machines, programming languages, operating systems, and other online resources may vary drastically. The term interoperability involves not only the ability of Internet GIS to interpret geospatial data in various formats, but also standard specifications describing how geospatial data should be encoded and documented to enable data exchange across the Internet (OGC 2007). The main benefit of specifications is that they enable a service-oriented model of data processing in which various geospatial services distributed on the Internet can interoperate with one another (Sondheim et al. 1999). The OGC is an international organization of software development companies, government agencies, and universities participating in a consensus process to develop publicly available interface specifications for geospatial data. Several OGC specifications for Internet GIS communication protocols, such as WMS, Web Feature Services, and Web Coverage Services, have been adopted recently by many Internet GIS software developers and are become more and more popular. 
Currently, WMS is one of the most acceptable communication protocols for Internet GIS. The WMS specification includes two mandatory interfaces (GetMap and GetCapability), and one optional interface (GetFeatureInfo). The GetCapability interface allows a Web map browser (client) to query and learn the capabilities of the map service provided by a Web map server. It can identify map layers, projections, image file format, and available symbolization options. The GetMap interface provides the connection for the Web map server to send back an actual request for a georeferenced map (picture). The third interface, GetFeatureInfo, allows a map client to send a pixel location back to the server to identify the location of the feature, and then to return the available attribute information about that feature (OGC 2007). The following string is an example of a GetMap specification from the TerraServer (source: http:// terraserver.microsoft.com/ogcwms.aspx, last accessed on July 27, 2007).

GetMap Layer: DOQ Style: UTM Grid Red SRS: EPSG: 26910 BBOX: 547,200.00, 4,182,400.00 zone 10: 553,600.00, 4,186,400.00 zone 10 Geographic BBOX: -122.463942, 37.787740: -122.390963, 37.823437 Width Resolution ( $m / p x)$ : 8.0000 Height Resolution ( $m / p x)$ : 8.0000 .

Another example of a popular GIS data standard is the Geography Markup Language (GML), which provides XML encoding for the transport and storage of geographic information. GML includes both the geometry and properties of geographic features. This specification defines the mechanisms and syntax that GML uses to encode geographic information in XML (OGC 2007). The following is a simplified example of a line feature described in GML.

The Geography Markup Language LineString Element Example:

$$
\begin{aligned}
& <\text { LineString srsName='EPSG:4326'> } \\
& <\text { coordinates }> \\
& 0.0,0.0 \\
& 30.0,50.0 \\
& 100.0,100.0 \\
& </ \text { coordinates }> \\
& </ \text { LineString }>
\end{aligned}
$$

Most Internet GISystems today adopt three-tier software architecture for system implementation (Figure 1; Tsou 2004). The first tier, called the client tier, includes the user-side Web browser and user-resident Java applets/HTML documents. The user of Internet GIS interacts with the client tier via a graphical user interface, comprised usually of a map and map navigation, spatial and attribute data query, and spatial analysis tools. The primary function of the client tier is to accept users' data requests and to display the results. The second tier, called the middleware tier, includes the Web server and the server connectors (such as servlet connectors or active server pages connectors), which bridge the communication between clients and the map servers. The third tier is the data 


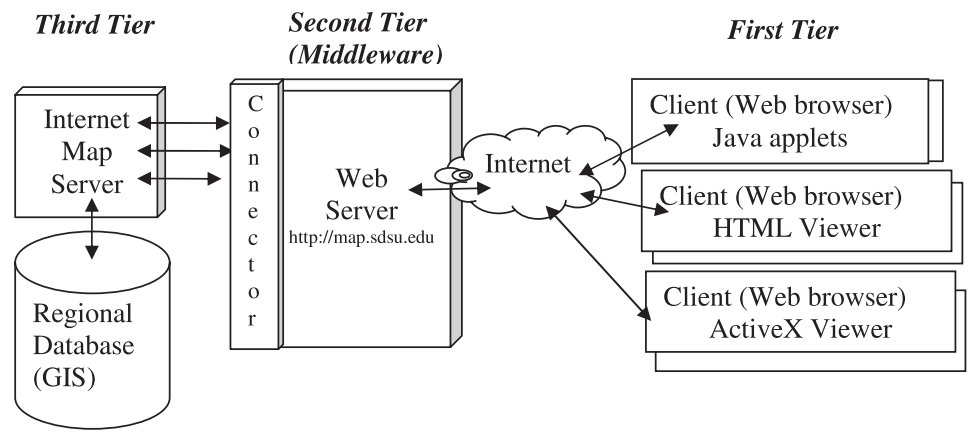

Fig. 1. The architecture of three-tier Internet GIS.

storage tier, which includes the map server and the spatial database server. The three-tier software architecture of Web-based GIS provides customizable functions for different mapping applications and scalable implementations for different volumes of use. It also provides correspondingly different hardware configurations.

There are many Internet GIS applications utilizing Web services and application development interfaces (API) for Web mapping or locating features based on their addresses (geocoding). Web services are interoperable and self-describing applications, which can communicate with each other. Many software companies such as ESRI, Google, Yahoo!, and Microsoft, have developed several Web service applications and Web Map APIs. Web application developers can combine and utilize these APIs and Web services into Web-based GIS applications that can provide basic mapping and GIS functions. It is likely that some current users of desktop GIS software packages will use exclusively Web-based GIS services in the future, and consequently the user base of desktop GIS will shrink. Already today, GIS users can conduct spatial operations such as map and feature attribute queries, geocoding, and map overlay by accessing remotely multiple Web services or APIs rather than using a single, centralized desktop GIS package. For example, a school bus driver can request a Web service to create the shortest route for the student transportation plan each month and adjust the route daily based on the traffic situation. The advantages of Web services include easy integration with other Web GIS applications and the low cost of software and hardware compared to the high cost of software and hardware required by traditional GISystems.

One major advantage of Internet GIS over traditional desktop GIS is the ability of the former to combine multiple map layers and GIS functions from multiple, heterogeneous Internet GIS servers. This offers a possibility of harnessing a tremendous computational power from vast computational resources connected to the Internet. The future development of Internet GIS will rely on establishing a comprehensive 
cyber-infrastructure framework that helps to organize all available distributed GIServices and Web servers on the Internet. One possible approach is to develop Web GIS portals (Tang and Selwood 2005), which utilize Grid computing technologies (Zhang and Tsou 2005), Web services (Tu and Abdelguerfi 2006), and Semantic Web technology (Berners-Lee et al. 2001). GIS users could use Web portals to analyze geospatial problems, find useful mapping services, request geospatial data, and aggregate GIS analysis results.

\section{Internet GIS and Public Participation}

With the maturation of technology and software tools and high penetration rates of the Internet into society $(70 \%$ of households in the United States had access to the Internet as of March 2007; source: www.internetworldstats.com/stats4.htm, last accessed on April 26, 2007), the potential of Internet GIS for delivering content relevant to the public has become a real possibility. Part of the content includes data and information about natural resources and public infrastructure such as roads, parks, waterfront facilities, and other public-use places. The idea that geospatial information content stored in GIS might empower different groups of the public including marginalized community groups gave rise to the concept of participatory GIS (PGIS) (Harris et al. 1995). In its original form, PGIS was conceived as a bottom-up, technology-facilitated, social process allowing users to utilize their informal and often qualitative knowledge, impressions and ideas, and combine them with formalized knowledge about real-world objects, their locations, and properties stored in GIS databases (Talen 2000). As various groups of participants develop interest, different tools, and participatory processes have grown to where today PGIS comprises a prominent area of research in GIScience using different, but related names such as public participation GIS (PPGIS), collaborative GIS (CGIS), group spatial decision support systems (GSDSS), and others (Balram and Dragicevic 2006; Sieber 2006). The interest in wider usage of geographic data and GIS has also spurred critical inquires into socially situated implications of GIS-based technology (Harvey et al. 2005).

The appeal of Internet GIS in service of open (democratic) decisionmaking processes is in potentially allowing the public to participate in the issues being discussed anywhere with Web access at any time. The range of participation supported by Internet GIS may cover a wide spectrum from informing to taking an active role in the formulation of decision options. Arnstein (1969) used the metaphor of a participation ladder comprised of eight rungs to demonstrate levels of participation. The levels start from 'non-participation' (manipulation, therapy) to 'tokenism' (informing, consultation, and placation) to finally 'citizen power' (partnership, delegated power, and citizen control). Arnstein's ladder metaphor has 
been used and modified in PPGIS context. For example, Schlossberg and Shuford (2004) theorized on why different groups within the public sphere when intersected with different types of participatory processes can pose different requirements for technological support including GIS. In the model presented by Schlossberg and Shuford, static and interactive Web pages, which one may be considered the products of Internet GIS, facilitate simple forms of participation corresponding to Arnstein's tokenism level. Peng (2001) argued that Internet GIS could potentially support public participation at any rung of the ladder through instant access to data and data-processing tools anywhere at any time. Because Internet GIS does not require specialized software, but usually only an access to the Internet, it provides in theory an open access to information. Steinmann et al. (2004) evaluated 12 studies of public participation involving the Internet as a platform for communication and dissemination of information. They discovered that a highly interactive citizen information exchange platform was the exception rather than the rule. They also observed that the efforts to involve citizens in participatory decision processes supported by Internet GIS were hampered not as much by technological shortcomings as by 'rational ignorance' of citizens, who were unwilling to spend time familiarizing themselves with data and tools unless they could see the benefit of doing so outweighing the effort of getting involved. The phenomenon of rational ignorance arises not only from the perception of an unfavorable cost-benefit ratio of getting involved, but also from the top-down organizational model of public participation, in which government organizations facilitate participation processes. These processes typically involve consultations with stakeholders on issues related to policy development in domains such as urban planning, resource administration, and environmental management. The effectiveness of the top-down model in broadening public participation has been mixed at best. There is also evidence that a bottom-up participatory process can be more effective at broadening public involvement than top-down processes (Craig et al. 2002; Owen et al. 2006; Talen 2000). New tools for participation and social networking in Web 2.0 such as blogs, wikis, RSS feeds, tagging, and mashups have become the enablers of bottom-up participatory processes. The challenge for designers of Internet GIS is to supplement Web 2.0 social networking tools with visualization and exploration tools that can capture the interest of the public, and get the members of the public involved. In the following sections, we present an example of Internet GIS design aimed at facilitating public involvement in water quality monitoring.

\section{The San Diego Common Ground: Water Quality Monitoring Portal}

Water quality influences quality of life, public health, and the local economy in the city of San Diego - a fact that is not lost upon its residents who, 
since 1999, have identified protection of recreational water quality as the highest priority for the city. The importance of regional water quality has been heightened by continued impacts on local streams, lagoons, and San Diego Bay. Since 1976, the number of impaired water bodies (not meeting federal standards) in the San Diego region has more than doubled with each subsequent development of the Clean Water Act, Section 303(d) list. The federal Clean Water Act, Section 303(d), requires states to identify water sources that do not meet certain water standards, and develop TMDLs for such waters (source: www.epa.gov/owow/tmdl/, last accessed on July 27, 2007). In California, the state government has developed a policy for the State Water Resources Control Board, with the Regional Water Control Boards to follow, to create and apply Section 303(d) lists. The process includes listing and delisting factors, procedures for gathering and evaluating data, and TMDLs scheduling. TMDLs are established for pollutants that exceed water quality objectives in water bodies that do not meet water qualities as set forth in Section 303(d) of the US Federal Clean Water Act. Numeric targets in TMDLs are determined and set at levels that are necessary to attain water quality objectives and compliance with water quality standards. The proposed 2006 Section 303(d) list for the San Diego Bay Watershed includes more than 20 segments that do not meet applicable water quality standards. Most of these segments are in San Diego Bay itself.

Despite the allocation of significant resources to monitoring the efforts put into place by the state government, there remains an inadequate understanding of local water quality, which undermines the ability to properly manage these vital resources. A great part of this problem is that while water quality and related monitoring efforts have been undertaken in the region for many years, there is at present no method to effectively manage and utilize data collected by regulatory agencies, academic institutions, businesses, and non-profit organizations. Inadequate data management hinders the ability of local and state agencies to arrive at informed management decisions to effectively identify and abate point and non-point source pollution. Water quality monitoring data must be obtained and effectively managed in order to protect sensitive ecosystems, identify and abate pollution sources, track the effectiveness of implemented actions, and prevent further degradation of our precious water resources.

With these water quality challenges facing the region, the San Diego Common Ground project aimed to incorporate data from water and sediment quality monitoring programs, and to integrate these data at a watershed scale using Internet GIS. The goal of the system has been to serve as a broad communication, education, and decision-making tool. Internet GIS would also develop the region's capacity to understand and access data about the processes affecting the area water resources. The San Diego Common Ground project started in the summer of 2004 through 
Proposition 13 grant funding from the California State Water Resources Control Board and ended in the winter of 2006.

San Diego Common Ground is a demonstration project that integrates data from water quality monitoring programs in the San Diego Bay Watersheds. It utilizes an interactive Internet GIS application as a broad communication, education, and decision-making tool. The project's primary goal has been to develop further the region's capacity to understand processes affecting water resources so that pollution sources can be readily identified and abated. A parallel goal has been the creation of a model 'node' for watershed data management that can be replicated and connected throughout the San Diego region.

\section{Design of Internet GIS for Water Quality Monitoring in San Diego Bay Watershed}

The primary design objective was to create a GIS that delivered water quality monitoring data over the Internet in a manner useful to both technical (biologists, analysts, academicians) and non-technical (policymakers, clean water advocates) stakeholders. The design strategy was guided by a survey of other Internet GIS systems (Table 1) and an analysis of users' information needs. The design of the Internet GIS focused on Web mapping and did not include spatial analysis and modeling functions due to the difficulty of addressing a wide range of analysis and modeling needs that vary from agency to agency. Consequently, the design goal was to develop a robust mapping/GIS infrastructure that would facilitate visualization, spatial and attribute query, and downloading of water quality data, as well as enable users to run their own analysis/modeling routines on that data.

\subsection{DESIGN ARCHITECTURE}

The Internet GIS prototype was developed by using off-the-shelf ESRI software (ArcIMS, ArcSDE, ArcGIS). The primary reason for choosing the commercial software over an open source software was that ESRI products are the defacto standard for GIS software worldwide and have a large user base. This would ensure reliability in software maintenance and services, reasonable costs, a wide range of supported data types, future technological advances, and a large pool of trained users. These advantages applied directly to San Diego Common Ground users because most, if not all, agencies in San Diego County already have ESRI software. Customization and third-party software were adopted into the system design to bridge the gaps between the system requirements and standard ESRI products, particularly in the design of Web mapping user interfaces and database download functions on the user/client side.

The Common Ground Internet GIS prototype adopted three-tier client/server system architecture. The three-tier architecture includes a 


\section{Table 1. Survey list of watershed management/water quality Web sites.}

\begin{tabular}{|c|c|c|}
\hline & Web site name & URL \\
\hline 1 & $\begin{array}{l}\text { Water Quality in Ireland (Ireland's } \\
\text { Environmental Protection Agency) } \\
\text { (online water quality map) }\end{array}$ & $\begin{array}{l}\text { www.epa.ie/rivermap/data/ } \\
\text { rivmaptop.html }\end{array}$ \\
\hline 2 & $\begin{array}{l}\text { CaSIL: the California Spatial } \\
\text { Information Library Interactive } \\
\text { Mapping (ArclMS-based) }\end{array}$ & $\begin{array}{l}\text { http://ims.gis.ca.gov/website/casil/ } \\
\text { datathemes/viewer.htm }\end{array}$ \\
\hline 3 & $\begin{array}{l}\text { Idaho Department of Environmental } \\
\text { Mapping: leaking underground storage } \\
\text { tank (sensitive information; removed } \\
\text { from the Web site after August 18, } \\
\text { 2004) }\end{array}$ & $\begin{array}{l}\text { http://mapserver.deq.state.id.us/ } \\
\text { Website/deqwaters/viewer.htm; } \\
\text { www.deq.state.id.us/map_gisgis.htm }\end{array}$ \\
\hline 4 & $\begin{array}{l}\text { The Central Coast Ambient Monitoring } \\
\text { Program }\end{array}$ & www.ccamp.org \\
\hline 5 & Heal the Bay & www.healthebay.org \\
\hline 6 & $\begin{array}{l}\text { San Diego Coastal Ocean Observing } \\
\text { System (Scripps Institute of } \\
\text { Oceanography) (Opportunity for } \\
\text { User Submitting Data: YES) }\end{array}$ & http://sdcoos.ucsd.edu \\
\hline 7 & $\begin{array}{l}\text { Project Clean Water (San Diego Area) } \\
\text { (Opportunity for User Submitting } \\
\text { Data: YES) }\end{array}$ & www.projectcleanwater.org/ \\
\hline 8 & $\begin{array}{l}\text { Coastal Water Quality Monitoring } \\
\text { Inventory }\end{array}$ & www.sfei.org/camp/ \\
\hline 9 & $\begin{array}{l}\text { Ventura County Ocean Water Quality } \\
\text { Monitoring Program }\end{array}$ & $\begin{array}{l}\text { www.ventura.org/envhealth/ } \\
\text { programs/ocean/ }\end{array}$ \\
\hline 10 & $\begin{array}{l}\text { Texas Watch: Water Quality Monitoring } \\
\text { Environmental Education Community } \\
\text { Action }\end{array}$ & www.texaswatch.geo.swt.edu \\
\hline 11 & San Francisco Estuary Institute & www.sfei.org/index.html \\
\hline 12 & St. Louis River - River Watch & www.fdl.cc.mn.us/ei/rw/ \\
\hline 13 & $\begin{array}{l}\text { University of Rhode Island Watershed } \\
\text { Watch Program }\end{array}$ & $\begin{array}{l}\text { www.uri.edu/ce/wq/ww/html/ } \\
\text { ww.html }\end{array}$ \\
\hline 14 & $\begin{array}{l}\text { Southern California Coastal Waters } \\
\text { Research Project }\end{array}$ & www.sccwrp.org \\
\hline 15 & $\begin{array}{l}\text { USGS National Water Quality Assessment } \\
\text { Program }\end{array}$ & water.usgs.gov/nawqa/ \\
\hline
\end{tabular}

client component (Web browser), a middleware (Web server), and a data server component (GIS database). The three-tier architecture can provide more flexible and customizable functions and tasks for Internet mapping services because the additional data server frees up the general Web server for more extensive processing. Figure 2 shows the three-tier architecture of the San Diego Common Ground Internet GIS prototype. This project uses two computer servers for delivering comprehensive Internet mapping services. One server www.trw.sdsu.edu) hosts the Web server and the Internet Map Server (ArcIMS). The second server www. geoinfo.sdsu.edu) 


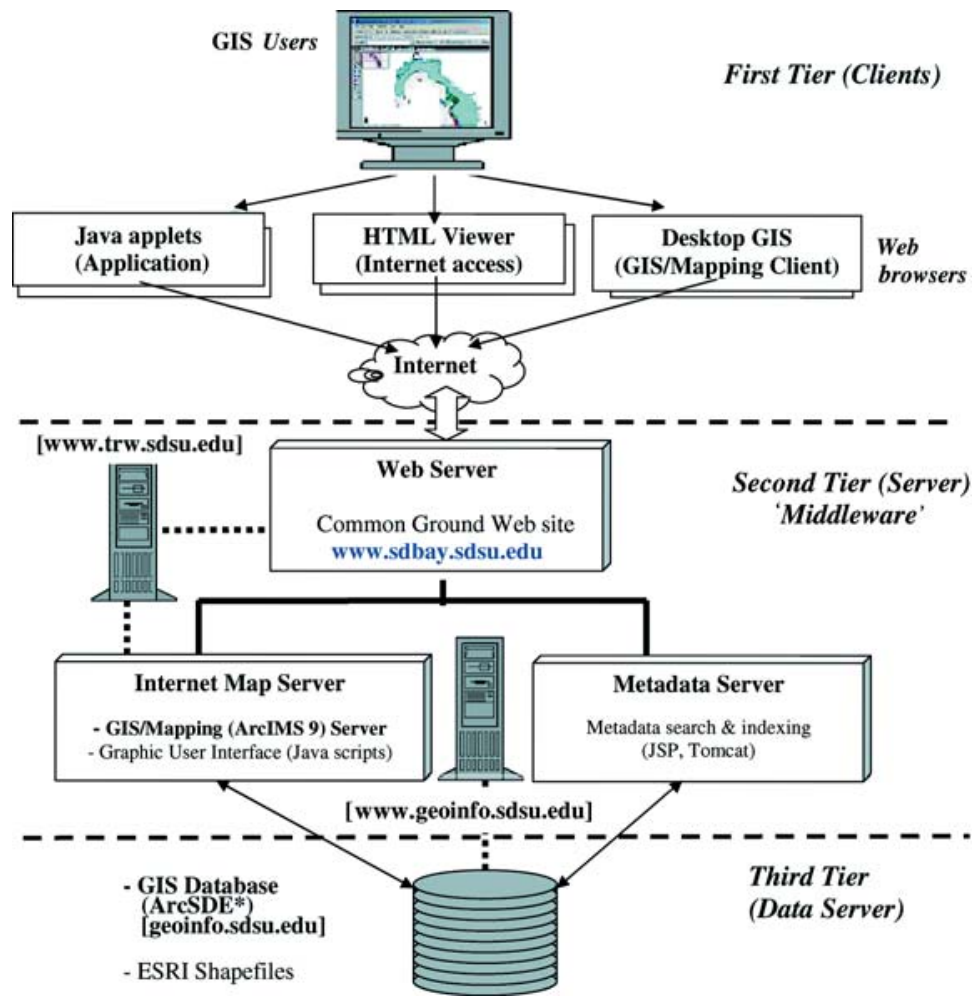

Fig. 2. The Three-Tier system architecture for the Common Ground Internet GIS prototype (clients, middleware, and data server).

hosts the metadata server and GIS databases. The Web server accesses GIS data and metadata from the metadata server.

\subsection{FUNCTIONAL CAPABILITIES OF THE SYSTEM}

The major functions of Internet GIS are accessible via the Common Ground project Web site (source: www.sdbay.sdsu.edu, last accessed on July 26, 2007), which can provide interactive, multifunctional information services functioning as a broad communication, education, and decision-making tool. The functionality of the Web site includes project descriptions, document archives, online geospatial mapping, spatial and attribute data query, and data download/sharing. As an outreach and educational tool, the Web site also includes important water quality concepts, news, statistical tables, graphs, and pictorials for a wide range of users.

There are three principal tools made available as a part of the project Web site, maps, database, and education. These tools appear as clickable 


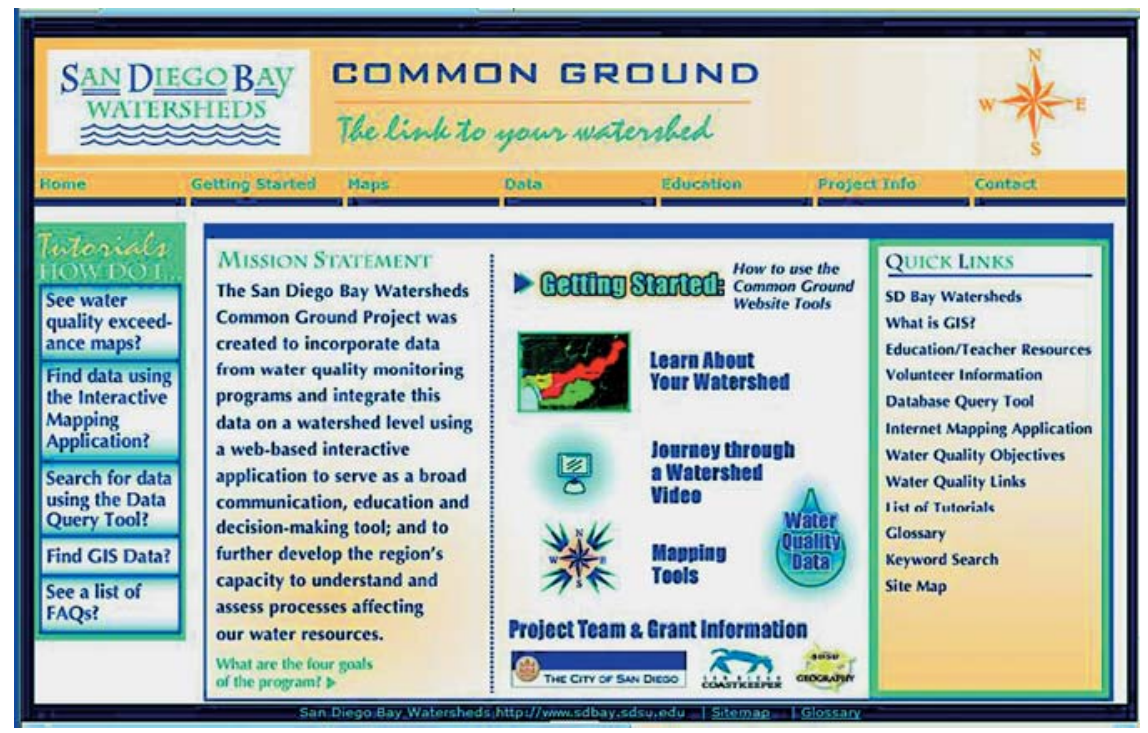

Fig. 3. The Common Ground project Web site (screen shot). There are seven drop-down menus on the top of the screen for accessing major functions (Home, Getting Started, Maps, Data, Education, Project Information, and Contact). The left-side window includes the shortcuts of GIS tutorials. The right-side window includes the quick links to some popular contents, such as education resources, volunteer information, water quality objects, etc.

buttons along the top of the page immediately beneath the Common Ground logo (Figure 3).

The Education Tool (www.sdbay.sdsu.edu/education/index.php) includes regional history, educator tools, a three-dimensional watershed video clip, and watershed science information.

The Database Query Tool (www.sdbay.sdsu.edu/data/index.php) allows the selection of water sampling results based on the parameter tested, location of sample, and watershed in which sample(s) were collected. Data can be queried by first agreeing to the disclaimer, which appears immediately after clicking the 'data' button located at the top of the Common Ground homepage. A table will appear with locations, samples, parameters, and output buttons along the top row. Clicking each button will open a new selection of criteria lists.

The Interactive Map Tools (www.sdbay.sdsu.edu/maps/index.php) include ArcIMS mapping services, PDF maps, Google Map mashup, and KML files for Google Earth. These tools allow users to query and access data using multiple maps and three-dimensional visualization tools.

The major interactive mapping tool of the Common Ground project runs as a customized application of ESRI ArcIMS software. Figure 4 illustrates a screenshot of the customized ArcIMS mapping services. The full extent view shows up once the map is accessed or when a user wants 


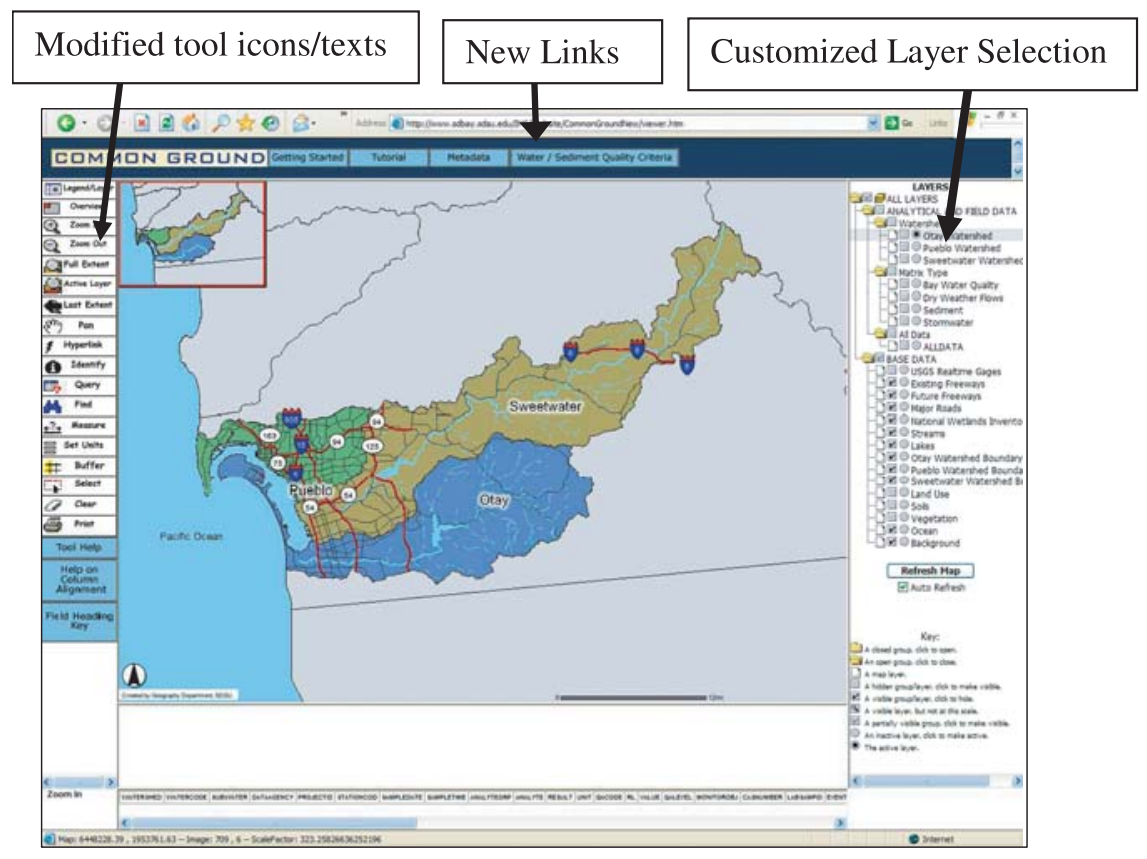

Fig. 4. The customized ArcIMS interactive mapping tool. Three major customization efforts are highlighted in this figure - the new tool icons with texts, the new functional link buttons, and the customized map layer section (with a hierarchical structure).

to return to the original view after using tools such as zoom or pan where the map view is changed. The LAYERS legend is on the right of the screen and the tools available for use are located on the left of the screen. The Map Window is in the middle of the page and shows the results of using the toolbar and the datasets. Along the bottom of the Map Window are the field headings for the results of the query tool. The toolbar is located to the left of the Map Window and contains functions for navigating and manipulating data within the Map Window. Tool help, help on column alignment, and the field-heading key are available by scrolling down the toolbar. The datasets are located in the scroll-down window to the right of the Map Window, and can be activated by selecting the circle or square buttons to the right of the specific dataset. There is a key section below the datasets, which details the functions and icon meanings found within the datasets. The ArcIMS complete tutorial is located at the top of the page. This comprehensive tutorial describes how to use all the functions of the interactive mapping tool. In addition, along the top of the page is the Getting Started link for the maps main page, a link to the metadata (descriptions of the different data and how the data was collected), and a link to the sediment and water quality criteria for the San Diego Bay Watersheds. 


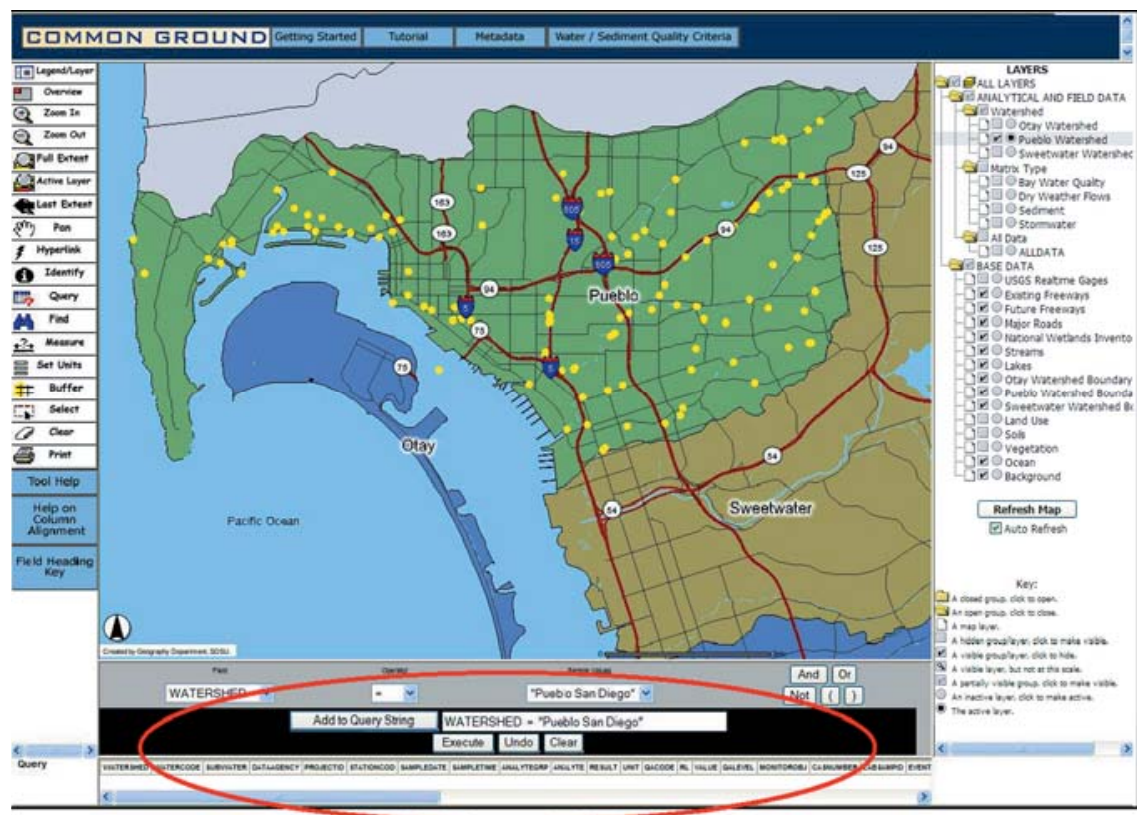

Fig. 5. The basic query function provided by ArclMS (screen shot). This query example illustrates the search for water quality monitoring sites within the Pueblo watershed boundary in San Diego. The query string can be generated automatically in the system and be executed online immediately.

The ArcIMS mapping tools provide various mapping functions, including zoom-in, zoom-out, pan, query, and identify. Figure 5 illustrates a screenshot of the query function after selecting Pueblo watershed as the spatial extent of query.

Besides the ESRI ArcIMS tools, the Web site also provides Google Map mashup tools for showing exceedance maps. These maps show when a parameter measured (such as a particular heavy metal - e.g. copper dissolved in water) exceeds the maximum contamination limit set by the State Water Resources Control Board. A red point on the exceedance maps indicates the violation of the water quality standard on the date that the parameter was measured. Hovering with the mouse over a point will give more information on the measurement at that point. An example of the exceedance map for Pueblo watershed bacteriological chemistry is presented in Figure 6.

Another tool of the Common Ground project provides the KML files for Google Earth Application allowing users to view maps in a threedimensional format. After downloading Google Earth KML files, users may zoom in on an area, visualizing the locations of the monitoring sites in a selected watershed. Figure 7 illustrated a screenshot of the 


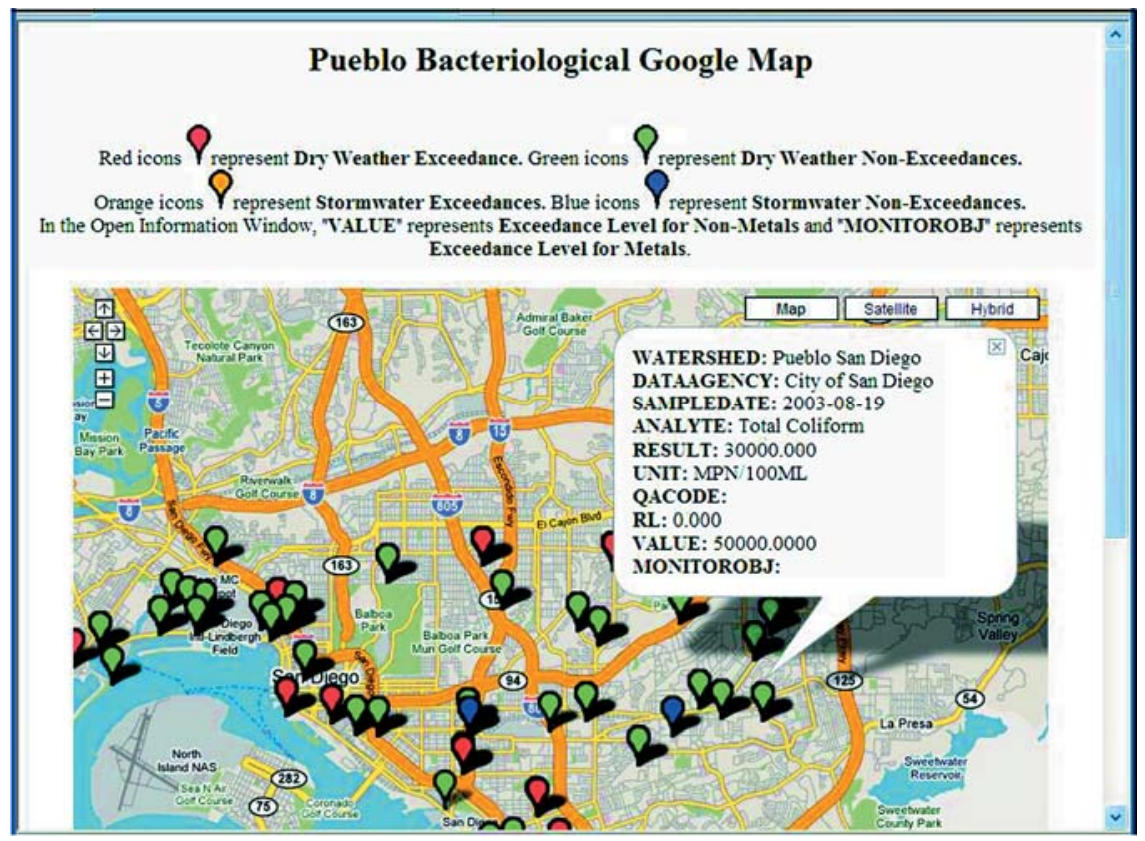

Fig. 6. The Google Map mashup map tool for water quality monitoring sites. The multiple point symbols shown on top of the Google Map are the locations of water quality monitoring sites. Each point contains detailed water quality information. When users click on the point symbols, detailed information will be displayed in a white text balloon window automatically.

water quality monitoring sites displayed in Google Earth by using KML files.

In summary, the Common Ground project adopted Internet GIS and Web mapping technologies to support the development of interactive watershed mapping tools. The project Web site aims to educate visitors with interactive mapping tools and online movies on various subjects pertaining to watershed hydrologic processes and water quality. The Web site also provided access to GIS datasets suitable for advanced spatial analysis and query functions. The intent of providing data, interactive, and educational multimedia tools has been to promote the awareness of the San Diego Watershed management problems and the understanding of the importance of water quality monitoring.

\section{Conclusion and Future Directions}

San Diego Common Ground combined many aspects of an applied research project in GIScience including education, discovery, and implementation within real-world users. In this final section, we reflect on lessons learned from the project and outline future developments. 


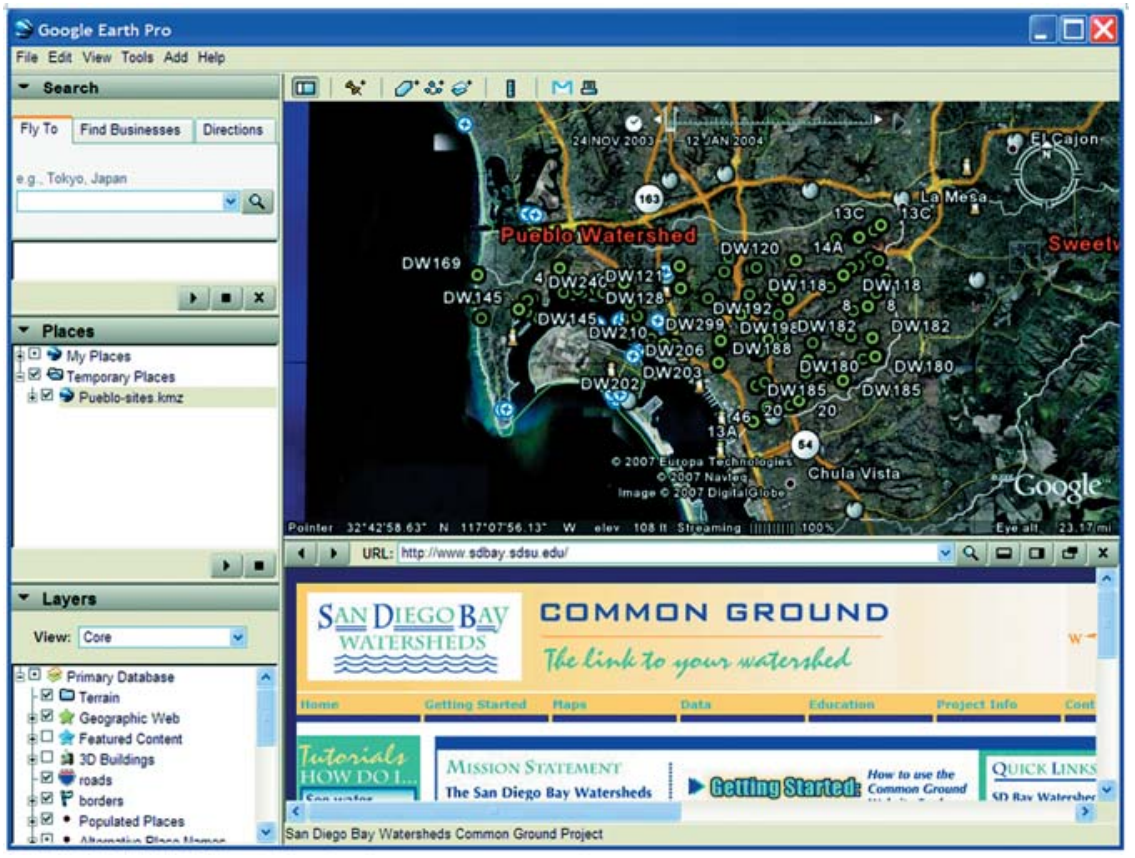

Fig. 7. Using KML files and Google Earth for the display of water quality monitoring sites. The green dots in the map windows are generated by adding a KMZ file (a compressed KML file) into the Google Earth application. The Google Earth application also provides a built-in Web browser at the bottom of the window (showing the Common Ground project Web site).

\subsection{LESSONS FROM THE DESIGN AND IMPLEMENTATION PROCESS}

One major lesson learned from the system design and implementation experiences has been the challenge of designing effective user interfaces for both the project Web site and the ArcIMS HTML map viewer. The major concern of Web site design is to create user friendly Web pages and navigation structures for the users. The project dealt with two different groups of target users (general public and GIS professionals) in order to provide effective guidance for the Web site structure. The Common Ground project Web site underwent revisions continuously through the duration of the project. The ArcIMS map viewer was also revised a number of times during the development of the project. The original ArcIMS HTML viewer was not user friendly and lacked a categorized map layers selection function. During a usability test of the viewer involving 10 users from San Diego the users had trouble with executing simple workflows. The test comprised of a short tutorial about the capabilities of the viewer and a 1-hr period of unrestricted viewer use during which user interactions with the viewer were first video-taped, and then discussed. Following the test results, a revised ArcIMS viewer added several new 
functions and online help tools. The new user interface has new graphic icons with text description for mapping tools. Most tasks of the customized ArcIMS now incorporate the user feedback from the usability test. The graphic design and iconic presentation play an important role for Web page layouts and ArcIMS mapping user interfaces. Based on the usability test, most users preferred graphic display and icon presentation rather than text-based descriptions. Another important lesson for Internet GIS design that we learned is the need for an iterative usability assessment during the course of system design. A spiral design approach, described in Jankowski et al. (2006) lends itself well to such an assessment by utilizing an iterative deployment, and testing consecutive versions of systems, accompanied by user feedback questionnaires.

Another challenge of developing Internet GIS applications is the accommodation of new Web technologies. Web technologies are continuously changing at a rapid pace (Peng and Tsou 2003). New Web technologies will have a significant impact on future developments of Web mapping applications. Future designers of Internet GIS applications requiring longer development times may face a hard choice between adopting a new technology in the course of system development and risking the technological obsolescence of a new system. Developers need to ensure that new Web technologies can fulfill the needs of users and mapping objectives. In some cases, new Web technologies could cause more problems in system usability if not properly evaluated. The additional iterative design processes for new Web technologies may generate a significant delay in a system's implementation and the release of Web mapping services.

\subsection{TOWARD DISTRIBUTED INTERNET GISERVICES}

Internet GIS-based systems such as Common Ground aim primarily at informing their users through access to data and information. In this sense, they rank low on the spectrum of participation levels, activities, and impacts (Nyerges et al. 2006). However, this is not to say that participatory processes beyond informing, such as public meetings or distributed, deliberative-analytic collaborations could not be supported by Internet GIS. An example of a state-of-the-art system (currently under development) based on technologies and concepts germane to Internet GIS, is Participatory Geographic Information System for Transportation (PGIST; source: www.pgist.org, last accessed on April 26, 2007). PGIST's goal is to support citizens willing to take time and familiarize themselves with transportation issues so that they can advise decision-makers on the selection of transportation infrastructure projects. PGIST will allow citizens with access to the Internet to discuss transportation-related concerns, match the concerns with evaluation criteria, evaluate various transportation projects, and prioritize these projects from the most to the least 
desirable. All of these features can provide decision-makers with a meaningful perspective on public priorities with regard to transportation improvement projects. The capabilities of PGIST will be available to the public through the PGIST Web portal developed as integrated Web services. Each major function of the portal is implemented as a service allowing the user to access it from any Web browser. Such design promotes the reusability of Web services, and allows their quick reconfiguration from one system into another to support a different type of participatory process or a different problem domain.

Future Internet GIS systems supporting various forms of collaborative work and participation will utilize the growing functionalities of geospatial Web services and service-based technologies. They will support various geographic decision processes ranging from closed collaborations involving experts distributed in space and tasked with solving a specific problem (e.g. various agency employees developing a water quality monitoring plan), to experts working with decision-makers and members of the public (e.g. in recommending projects for a transportation improvement program). They will also support various models of collaboration. One such model called analytic-deliberative, prototyped in the PGIST project, uses an online analytic component (maps linked with graphs, tables, and simulations) to help participants enhance their competence of analytic knowledge. The deliberative component enables the participants to enhance their access to online voices for synthesizing interpretations of analytic knowledge. Models such as analytic-deliberative that can guide the use of Internet GIS tools offer the potential for a meaningful participatory problem solving and decision-making.

\section{Short Biographies}

Dr. Piotr Jankowski received PhD in Geography from the University of Washington and MS in Econometrics and Operations Research from the Poznan School of Economics. He held faculty appointments in Geography at the University of Idaho and in Geoinformatics at Muenster University in Germany. He is currently a professor in the Department of Geography at San Diego State University. His research areas include Spatial Decision Support Systems and Participatory GIS with applications in Water Resources and Transportation. He is the author of over 60 publications on multiple criteria decision-making methods, spatial decision support systems and spatial modeling.

Dr. Ming-Hsiang (Ming) Tsou is an Associate Professor in the Department of Geography, San Diego State University. He received a BS from National Taiwan University in 1991, an MA from the State University of New York at Buffalo in 1996, and a PhD from the University of Colorado at Boulder in 2001, all in Geography. His research interests are in Internet mapping and distributed GIS applications, mobile GIS and wireless 
communication, multimedia cartography and user interface design, and software agents with Grid computing technology. He has applied his research interests in applications such as wildfire mapping, environmental monitoring and management, habitat conservation, and homeland border security. He is co-author of the book, Internet GIS: distributed geographic information services for the Internet and wireless networks. Dr. Tsou is the co-chair of the NASA Earth Science Enterprise Data System Working Group (ESEDWG) Standard Process Group (SPG) from 2004 to present.

Dr. Richard D. Wright is Emeritus Professor of Geography and Past Co-Director of the Center for Earth Systems Analysis Research at San Diego State University. Dr. Wright holds a PhD in Geography from the University of Kansas (1967), an MA in Geography from Indiana University (1961), and a BA in Geology from Indiana University (1960). He is a specialist on geographic information systems (GIS), cartographic visualization, and coastal watershed analysis. He has been a consultant to private industry and public agencies on a variety of mapping and geographic information systems projects. During the past 15 years, he has been engaged in GIS education and the use of GIS in land use and water quality modeling. Since 1987, he has been the PI or Co-PI of 85 awards, grants, and contracts from federal, state, and local agencies that have resulted in more than 100 research papers and professional presentations on GIS and related topics.

\section{Acknowledgements}

The Common Ground project was funded in part by the California State Water Resources Control Board and the San Diego Foundation. The support of these organizations in carrying out the project is gratefully acknowledged. We would also like to thank two anonymous reviewers whose comments helped us to strengthen the manuscript.

\section{Note}

* Correspondence address: Piotr Jankowski, Department of Geography, San Diego State University, 5500 Campanile Drive, San Diego, CA 92182-4493, USA. E-mail: piotr@geography.sdsu.edu.

\section{References}

Arnstein, S. (1969). A ladder of citizen participation. Journal of the American Institute of Planners July, pp. 216-224.

Balram, S., and Dragicevic, S. (eds) (2006). Collaborative geographic information systems. New York: Idea Group Inc.

Berners-Lee, T., Hendler, J., and Lassila, O. (2001). The semantic web: a new form of web content that is meaningful to computers will unleash a revolution of new possibilities. Scientific American 284 (5), pp. 34-43. 
Butler, D. (2006). Virtual globes: the web-wide world. Nature 439, pp. 776-778.

Buttenfield, B. P., and Goodchild, M. F. (1996). The Alexandria Digital Library Project: distributed library services for spatially referenced data. Proceedings of GIS/LIS '96, Denver, Colorado, November 19-21, 1996, pp. 76-84.

Craig, W. J., Harris, T. M., and Weiner, D. (eds) (2002). Community participation and geographic information systems. London: Taylor \& Francis.

Green, D., and Bossomaier, T. (2002). Online GIS and spatial metadata. London: Taylor \& Francis.

Harris, T., et al. (1995). Pursuing social goals through participatory geographic information systems: redressing South Africa's historical political ecology. In: Pickles, J. (ed.) Ground truth: the social implications of geographic information systems. New York: Guilford, pp. 196-222.

Harvey, F., Kwan, M.-P., and Pavlovskaya, M. (2005). Editorial introduction. Cartographica 40 (4), pp. 1-4.

Huang, B., and Worboys, M. F. (2001). Dynamic modelling and visualization on the internet. Transactions in GIS 5 (2), pp. 131-139.

Huse, S. M. (1995). GRASSLinks: a new model for spatial information access in environmental planning. Unpublished doctoral dissertation, University of California, Berkeley, CA, USA.

Jankowski, P. et al. (2006). Design considerations and evaluation of a collaborative, spatiotemporal decision support systems. Transactions in GIS 10 (3), pp. 335-354.

Nyerges, T., et al. (2006). Collaborative water resource decision support: results of a field experiment. Annals of the Association of American Geographers 96 (4), pp. 699-725.

Open Geospatial Consortium (OGC). (2007). OpenGIS specifications (standards). [online]. Retrieved on 26 April 2007 from http://www.opengeospatial.org/standards

Owen, A., Jankowski, P., and Williams, B. (2006). Spatial data for water resource protection: field study on a North-central Indian idaho reservation. Journal of Environmental Assessment Policy and Management 8 (4), pp. 431-450.

Peng, Z. R. (2001). Internet GIS for public participation. Environment and Planning B: Planning and Design 28 (6), pp. 889-905.

Peng, Z. R., and Tsou, M. H. (2003). Internet GIS: distributed geographic information services for the internet and wireless networks. New York: John Wiley \& Sons.

Peterson, M. P. (ed.). (2003). Maps and the internet. Oxford, UK: Elsevier Press, Published on behalf of the International Cartographic Association.

Plewe, B. (1997). GIS online: information retrieval, mapping, and the internet. Santa Fe, NM: OnWord Press.

Putz, S. (1994). Interactive information services using World Wide Web hypertext. In: Proceedings of the first international conference on the World Wide Web. Geneva, Switzerland. [online]. Retrieved on 5 November 2006 from http://www94.web.cern.ch/WWW94/ prelimprocs.html

Schlossberg, M., and Shuford, E. (2005). Delineating 'public' and 'participation' in PPGIS. URISA Journal 16 (2), pp. 15-26.

Tu, S., and Abdelguerfi, M. (2006). Web services for geographic information systems. IEEE Internet Computing September/October, pp. 13-15.

Sieber, R. (2006). Public participation geographic information systems: a literature review and framework. Annals of the Association of American Geographers 96 (3), pp. 491-507.

Sondheim, M., Gardels, K., and Buehler, K. (1999). GIS interoperability. In: Longley, P. A., et al. (eds) Geographical information systems: principles and technical issues (Vol. 1, 2nd ed.). New York: John Wiley \& Sons.

Steinmann, R., Krek, A., and Blaschke, T. (2004). Analysis of online public participatory GIS applications with respect to the differences in the US and Europe. Proceedings of Urban Data Management Symposium '04, Chioggia, Italy.

Talen, E. (2000). Bottom-up GIS: a new tool for individual and group expression in participatory planning. Journal of the American Planning Association 66 (3), pp. 279-294.

Tang, W., and Selwood, J. (2005). Spatial portals: gateways to geographic information. Redlands, CA: ESRI Press.

Tsou, M. H. (2004). Integrating web-based GIS and on-line remote sensing facilities for environmental monitoring and management. In special issue on the potential of Web-based GIS. Journal of Geographical Systems 6 (2), pp. 155-174. 
Vckovski, A. (1998). Interoperable and distributed processing in GIS. London: Taylor \& Francis.

Yang, C., et al. (2005). Performance-improving techniques in web-based GIS. International Journal of Geographical Information Science 19 (3), pp. 319-342.

Zhang, T., and Tsou, M. H. (2005). The Integration of grid-enabled internet GIServices and geographic semantic web technologies. In Special Issue on Distributed GIS. Geographic Information Science 11 (1), pp. 15-23. 